Quaderni di Geografia Cahiers de Gécongaty rataphie

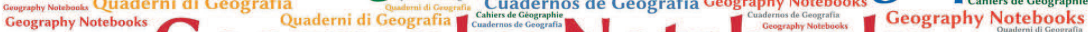
Cuadernos de Geografia Geography Notebooks $U 201000$ Cahiers de Géographi Cahiers de Géographie cahiers de Géographie Cuadernos de Geografía Cahiers de Géographie Cahiers de Géographie Cuadernos de Geografía Geography Notebooks

\author{
$4(2021)$ \\ 1 \\ Teatro di suoni. \\ Spazi acustici teatrali e territoriali
}

A cura di

Martino Mocchi, Lorena Rocca, Demis Quadri and Carlotta Sillano

EDITORIAL

Teatro di suoni per l'attaccamento ai luoghi. Uno sguardo geografico 11

Lorena Rocca

Per un teatro di suoni. Riflessioni su possibili dimensioni sonore nelle 23 creazioni site-specific di physical theatre

Demis Quadri

INTRODUCTION

Teatro di suoni. Spazi acustici teatrali e territoriali

Demis Quadri e Lorena Rocca

SPECIAL Issue

I suoni di Mantova come strumenti di interpretazione del paesaggio.

Tra turismo sostenibile ed educazione al patrimonio culturale

Valeria Pecorelli, Franca Zuccoli, Alessandra De Nicola, Enrico Squarcina

Il paesaggio sonoro campano tra contemporaneità e nuove forme

di progettualità turistica

Germana Citarella 
La narrazione spettacolarizzata del paesaggio sonoro.

Da Giuseppe Chiari a Philip K. Dick e oltre

Francesco Michi

Musica di paesaggi sonori. Enunciazione, risignificazione, comunicazione

Carlotta Sillano

Camminare per ascoltare. Partiture invisibili del territorio abitato

Elisabetta Senesi

Il paesaggio sonoro in relazione. Suono, movimento e immagini per stimolare complessità percettiva Angela Calia

Groove Fields. Understanding the Dance Floor from an Art-Based Research Perspective

Sebastian Mattbias

Il silenzio come esperienza trasformativa. L'importanza del silenzio nella meditazione e in ambito professionale

Sebastiano Caroni

Progettare il silenzio. Una lettura acustica dell'ex villaggio sanatoriale 125 Morelli a Sondalo

Martino Mocchi

Voicing One's Will. Theatre as Audio-Visual Hypotyposis of the Poetic

Michael Groneberg

Music and Clowning in Europe, 20th-21st centuries

Anna Stoll Knecht

Il paesaggio sonoro nella composizione musicale. Un percorso bibliografico

Stefano Alessandretti

\#exploreART: il labirinto di A. Pomodoro e i bambini. Un progetto di fruizione condivisa con percorsi sensoriali partecipati Alessandra De Nicola, Franca Zuccoli 


\section{OTHER EXPLORATIONS}

Il rumore lontano. Intervista a Lorena Rocca

a cura di Martino Mocchi

Re Cervo. Intervista a Antonella Astolfi

a cura di Krizia Bonaudo e Demis Quadri

Centovalli-Centoricordi. Intervista a Oliviero Giovannoni a cura di Krizia Bonaudo e Demis Quadri

Alla ricerca di un metodo: Open Space Technology 



\title{
Camminare per ascoltare. Partiture invisibili del territorio abitato
}

\author{
Elisabetta Senesi \\ Artista sonora e visiva \\ DOI: https://doi.org/10.7358/gn-2021-001-sene
}

\begin{abstract}
The paper is focused on some experiences of collective soundwalks achieved by Elisabetta Senesi within her conscious Sonic Arts research (MA Dissertation 2010). Walks are mostly performed in silence at different times and unpredictable urban paths for participants. This practice, not always performed following the tradition of Acoustic Ecology, has become particularly important in recent years, and often carried by artistic fields related to the creation of environmental compositions, both generative and interactive. In this context, Senesi uses her listening walks as case studies to open up multiple perspectives of space and critical listening, as well as its auditive aesthetics to reflect upon author's soundwalking strategies with feedback from participant's experiences from the last six years.
\end{abstract}

Keywords: walking; public space; critical listening; experimental theatre.

Parole chiave: camminare; spazio pubblico; ascolto critico; teatro-ricerca.

\section{INTRODUZIONE}

Fin dai primi modelli di passeggiata sviluppata negli anni Settanta dal "World Soundscape Project" (Schafer 1970), orientati verso una pedagogia e resistenza all'ascolto del design urbano, e quelle di Max Neuhaus (Kotz 2009), focalizzati sull'estetica e le prestazioni artistiche, questo ap- 
proccio uditivo allo spazio del quotidiano continua a sorprendere artisti, attori, musicisti, ricercatori, antropologi, sociologi, geografi, architetti, storici, talvolta sviluppatori informatici, e non addetti ai lavori. Nella sua forma più classica di soundwalk, un gruppo di persone viene coinvolto per concentrarsi sull'ascolto dello spazio circostante e, guidato in silenzio all'interno di uno o più ambienti quotidiani, percorre un tracciato predefinito. Oggi la disciplina del soundwalking viene utilizzata sempre più come forma attiva di lavoro sul campo sia dalle scienze sociali che all'interno di ricerche affini riguardanti gli studi del paesaggio sonoro e il controllo del rumore ambientale: un metodo di rilevamento del paesaggio in situ e raccolta dati analitica, ma trova anche spazio nel territorio della composizione artistica e musicale apportando molti temi insiti quali la partecipazione, il contesto sociale, l'ascolto estetico, una certa sensibilizzazione ambientale, l'interpretazione dei luoghi, la pedagogia uditiva, un innalzamento della consapevolezza, fino a riflessioni psicogeografiche e catartiche.

I fisici acustici hanno elaborato e perfezionato industrialmente criteri per misurare e prevedere la percezione umana e la risposta al rumore ambientale come i valori $\mathrm{dB}$ e affini, ma mancano molti parametri quando si tratta di tracciare le vicissitudini dell'ascolto del quotidiano. Il metodo apparentemente non empirico del soundwalking, al contrario, può suscitare qualcosa nell'interrelazione umana con il territorio e i luoghi di tutti i giorni, siano essi affettivi, valutativi, strategici, abituali, semantici o estetici. Questo, infatti, può essere praticato in un momento non specificato da chiunque non sia ostacolato da problemi di accesso o mobilità e, come qualsiasi disciplina, può essere sviluppata e interpretata. Nella sua accezione più classica legata alla tradizione artistica nell'ambito dell'ecologia acustica, si tratta dello sviluppo di percorsi uditivi in cui è richiesto un ascolto attento e pronto, privo di pregiudizi, che si protrae al di là delle mura dei siti musicali deputati (ad es. sala da concerto, teatro) all'interno di una forma comune di passeggiata. In tale definizione, tuttavia, le motivazioni, le metodologie e le manifestazioni/rappresentazioni variano radicalmente rispetto al contesto in cui avvengono (assolo o ensemble). Al contrario ed evitando prescrizioni predeterminate, può assumere la forma di una performance estemporanea di gruppo, in base alle realtà che l'ambiente circostante offre, improvvisando in corrispondenza con le ansie dell'artista, come nel caso delle camminate che vengono realizzate e condotte a vari livelli di ascolto.

Il Soundwalking è qualcosa che gli artisti hanno abitualmente esegui- 
to senza un codice temporale ben definito, e come tale si trova a cavallo in quella zona grigia tra il lavoro sul campo e la pratica artistica (Kelly, C. 2012). Qualunque sia il livello di determinazione e di tecnologia mediata (che almeno richiede un buon paio di scarpe da passeggio), i ruoli gerarchici di compositore, esecutore e pubblico sono spesso indivisibili, e in qualsiasi contesto la paternità del lavoro è quasi sempre problematica. Come andremo ad analizzare nel prossimo paragrafo, la passeggiata silenziosa pone senza dubbio delle domande sul nostro impegno verso il mondo, focalizzandolo nella sensibilità uditiva individuale e collettiva. Questa attività richiede una attenzione non lontana da un ascolto in una sala da concerto o teatro, ma ci troviamo fisicamente collocati e attraversiamo lo spazio del quotidiano: uno stato che stimola naturalmente il comportamento, e che è in contrasto con le contingenze di ascolto di una sala da concerto o teatro. Così come il discernere tra segnale o rumore, tra primo piano e sfondo acustico, tra estetica e funzione; tale approccio, come vedremo, diventa anche una questione di atteggiamenti e sensibilità sempre mutevoli.

\section{Camminare come pratica di ASCOlto}

Il compositore/conduttore che si esprime con la passeggiata di ascolto in una prospettiva artistica fornisce delle modalità inclusive verso e per un territorio specifico, analizzandone punti forti e criticità attraverso un costrutto teorico fondato, come accade ad esempio nel lavoro dell'artista performer sonora brasiliana Vivian Caccuri, in cui le persone vengono condotte in un cammino silenzioso urbano di otto ore (2015). Inoltre, poiché camminare è il mezzo e l'azione fisica del soundwalking, il capitolo espone alcune incursioni effettuate dall'autrice in ambito per lo più pubblico, dove il comportamento pedonale diventa metafora di una cultura radicale contemporanea, contribuendo a dare credibilità a tale pratica. L'incedere dei passi che avanzano su diverse superfici geometriche, così come l'andatura e cadenza del camminare, è una caratteristica della vita quotidiana di quasi tutti noi, per molti è un mezzo di trasporto immediato e primario oltre che di conoscenza del mondo. Questo ci porta a considerare l'aspetto esperienziale dei corpi all'interno di un teatro di suoni e rumori che restano ai più inascoltati. Camminare a orecchie tese può inoltre essere un'attività altamente sociale, sana, piacevole e apparentemente senza utilizzo di materie inquinanti. 
I passi formano anche un percorso variabile di trasmissione per la vibrazione tra la superficie stessa (cioè il suolo) e il corpo, anche se interrotto a intermittenza dalla fase in cui si trova l'andatura del gruppo. Inoltre, in quanto esseri multisensoriali, anche gli altri sensi quali l'olfatto e la vista interagiscono con lo spazio di ascolto all'interno di logiche comportamentali dettate da relazioni inedite, e paragoni acustici precedentemente ignorati. Come ad esempio cogliere una sintonia tra suono ed ambiente, oppure il suo odore, fermarsi ad ascoltare la voce delle cose, il muoversi all'interno di un flusso costante tra interno ed esterno dove sensazione, percezione ed attenzione coabitano. In una camminata realizzata nella parte più antica della città medievale di Siena con un gruppo numeroso ed eterogeneo per età, estrazione e genere, è stato interessante scoprire come da artista conduttrice la fase iniziale di esplorazione, introduzione e guida fosse cruciale alla buona esecuzione del lavoro, soprattutto laddove gli spazi erano molto compatti e i partecipanti non sapevano come muoversi, o che modalità di ascolto adottare in una città per lo più impenetrabile (Fig. 01).

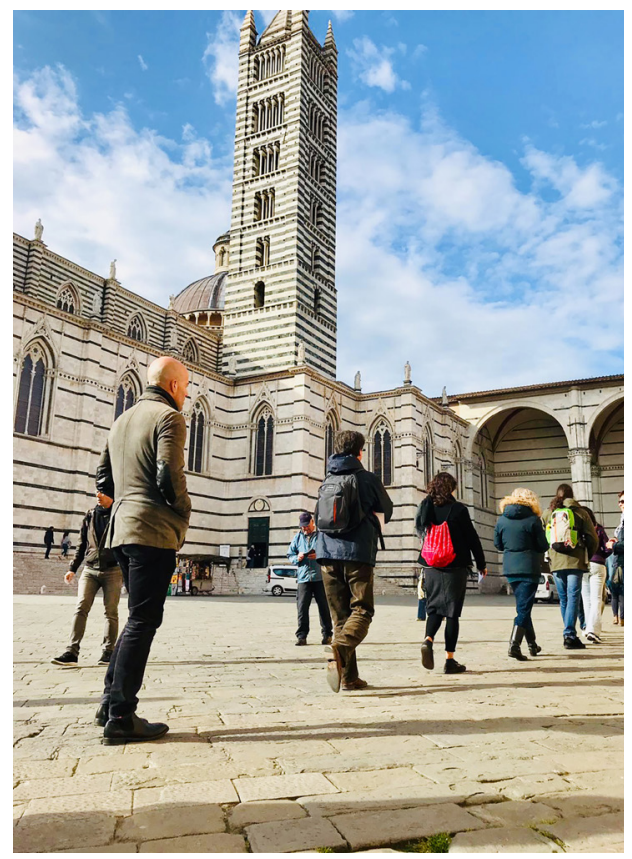

Figura 1. - Soundwalk a Siena nel maggio 2019.

Fonte: fotografia di Irene Lupi per SART Siena Art Institute.

Geography Notebooks - 4 (2021) 1

https://www.ledonline.it/Geography-Notebooks/ 
Nonostante l'ubiquità e l'universalità del camminare, per il pedone l'esperienza urbana può anche essere incisivamente ostile e alienante, a causa di strade particolarmente rumorose, inquinate, spiacevoli o per lo più progettate a misura di automobile. Tale può essere il contesto per la soundwalk urbana che è concepita su scala umana del pedone in strada e/o percorso, includendo vari luoghi quali giardini pubblici, piazze di quartiere, alloggi in costruzione, centri commerciali, siti industriali dismessi; ma è in tale contesto scenico pubblico che le mie passeggiate di ascolto si inseriscono e si impegnano.

\subsection{Metodologia}

Tenendo conto delle questioni pratiche che tendono a influenzare una camminata (scala temporale, punti di inizio e fine, abilità dei camminatori, programmi dei partecipanti, condizioni meteorologiche ecc.), rimane un protocollo efficace quello stabilito dal World Soundscape Project guidato da Murray Schafer nei primi anni Settanta, e che in parte applico. Una volta che il gruppo si è riunito, l'artista conduttore prepara la scena e i suoi camminatori. Ciò potrebbe includere una discussione sui metodi di ascolto, l'anticipazione di ciò che si incontrerà considerando la geografia e i tempi e un promemoria di problemi di salute e sicurezza, in particolare relativi al traffico (i partecipanti sono incoraggiati a riflettere su un comportamento sensoriale implicito, ma ciò può renderli vulnerabili ai pericoli della strada). Ė importante stabilire un contratto affidabile tra il gruppo e il leader artista prima della partenza, al fine di creare un corpo di ascolto coeso che muovendosi lento nello scenario pubblico urbano acquista valore simbolico e scenico, coinvolgendo e problematizzando la realtà circostante.

Successivamente, il gruppo è invitato ad adottare un numero di regole: fare voto di silenzio per tutta la durata della passeggiata, telefono cellulare spento, nessuna registrazione audio-video della camminata, e camminare in una sola linea seguendo il leader. Mentre le prime tre regole sono una pratica comune per un pubblico di concerti di musica classica o teatro, la regola finale impedisce al singolo camminatore di avere effetti negativi sull'affollamento, e permette di creare un corpo sociale di ascolto che lentamente invade lo spazio. Personalmente invito a prendere appunti o disegnare attraverso una lista di domande e osservazioni redatta in precedenza, anche al fine di orientare i partecipanti a questo tipo di ascolto e tenere una traccia scritta di quanto incontreranno nel tragitto. 
Sebbene tale facoltà sia lasciata libera di essere seguita o ignorata, i camminatori possono inoltre decidere di fotografare entro perimetri limitati di ascolto in cui il gruppo viene lentamente decomposto attraverso un gesto del conduttore, stazionando individualmente per alcuni minuti in punti specifici, prima di ricomporsi e procedere nel percorso uditivo.

Il silenzio è parte integrante del lavoro, e più precisamente senza il silenzio verbale dei partecipanti non si verrebbe a creare quel movimento di corpi in ascolto sul mondo. Inoltre, tale esercizio e fatica del muoversi lentamente senza parlare induce a riflettere sulla qualità della relazione con il mondo stesso, e sulle persone che lo abitano. Questo implica un approccio di ascolto disciplinato, intenso e concentrato, oserei dire solenne e profondo, ma allo stesso tempo immaginativo ed errante che riflette sul proprio stare al mondo, ma anche su ciò che un suono/rumore può o non può significare all'interno di un territorio specifico. Ci possono essere momenti di affollamento agli incroci o agli attraversamenti pedonali, ma il gruppo può ripartire rapidamente, come in un concerto.

Ogni volta che guido un gruppo, sono immediatamente consapevole del camminatore dietro di me: i passi possono fornire uno strumento di riferimento efficace. Il cammino è tenuto a un ritmo insolitamente lento, ad esempio se una passeggiata viaggia su una scala mobile, il gruppo viaggerebbe alla velocità data. Avanzare lentamente è utile per aiutare a spostare le persone dalle pratiche abituali di ascolto o di non ascolto, e allo stesso modo consente alle persone di prendersi semplicemente il loro tempo. Ciò che a me interessa è che si creino delle micro-politiche del quotidiano, attraverso brevi e sottili spettacoli acustici il più trasversali possibili, come accaduto nelle ultime passeggiate realizzate a Firenze all'interno del grande parco urbano delle Cascine in cui coesistono realtà sociali molteplici (Fig. 02).

La camminata lenta e la contemplazione vanno di pari passo, ma i camminatori devono ricordare a sé stessi che se stanno diventando troppo introspettivi nel loro ascolto devono riconnettersi col gruppo, e non dimenticare il fatto di essere un corpo unico dinamico. La natura dell'esperienza del gruppo attento si ricollega nuovamente all'esperienza di una esibizione musicale o teatrale ritualistica, ma che può generare un'esperienza di ascolto intensificata e più estesa. Il gruppo fornisce anche supporto reciproco, aiutando a mantenere la disciplina dell'ascolto focalizzato. Il ruolo del leader è fondamentale, assumendo tutte le preoccupazioni quotidiane di cronometraggio e navigazione, lasciando ai camminatori silenziosi il lusso senza precedenti di concentrarsi esclusivamente sull'a- 


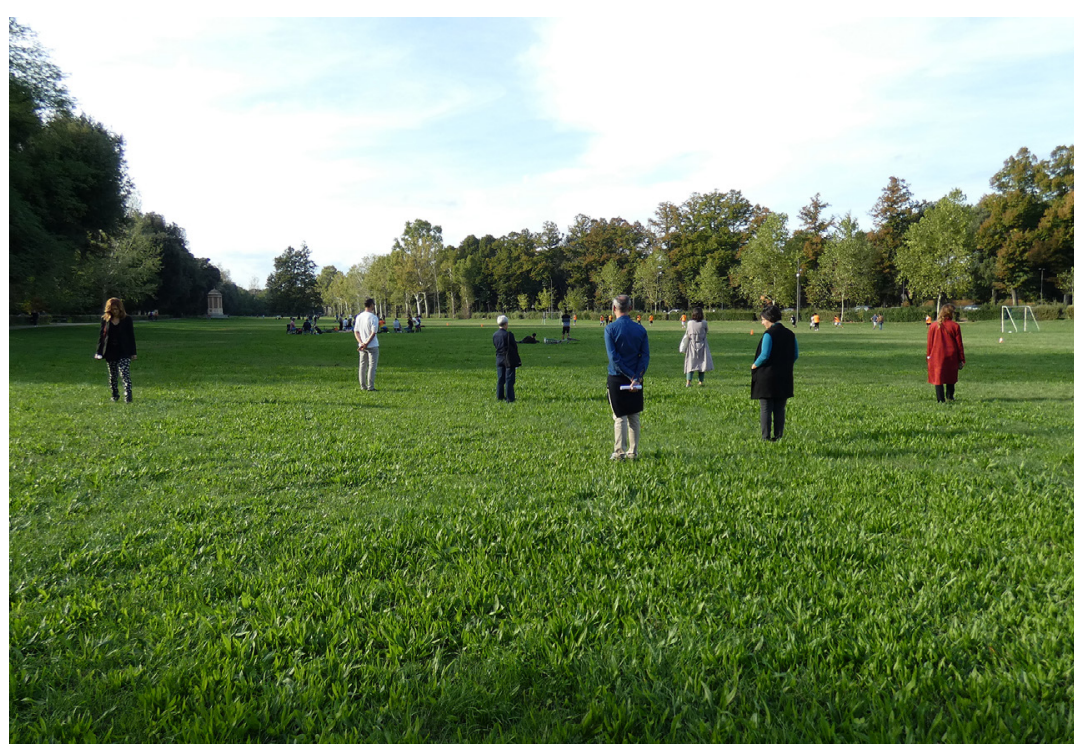

Figura 2. - Soundwalk realizzata a Firenze per Sonic Somatic, novembre 2019. Fonte: fotografia di Sonic Somatic.

scolto: una facoltà che è alla base, ma a causa delle pressioni quotidiane viene raramente esercitato per una durata significativa.

Le soundwalk che realizzo hanno una durata di minimo 30 minuti fino a un massimo di 2 ore, ovviamente vi è un forte aspetto performativo in un gruppo di persone silenziose che camminano lentamente per strada come fossero un enorme coccodrillo che si snoda lungo le vie della città. Questo può essere considerato vantaggioso o problematico: è meno probabile che una soundwalk troppo esplicita trasformi l'ambiente circostante in qualcosa di altro, mentre un percorso più ambiguo e diversificato può influenzare relazioni diverse in contesti diversi. Inoltre, in molte zone del centro città ci sono restrizioni legali per le attività di gruppo, a determinati punti che scelgo con molta accuratezza e sopralluoghi, il gruppo può fermarsi momentaneamente per raggrupparsi ed elencare sistematicamente rumori e sensazioni. Il silenzio è rotto alla fine della passeggiata con varie modalità verbali, mentre il gruppo è fermo sul posto i partecipanti sono incoraggiati a condividere apertamente le loro esperienze, pensieri e preoccupazioni. In tale fase, il soundwalking manifesta tutta la sua complessità di definizione e talvolta una certa ambiguità nella motivazione iniziale, quando i partecipanti sono invitati a verbaliz- 
zare la loro esperienza durante una discussione di sintesi nel momento di rottura finale.

\subsection{Itinerario}

Il percorso, pianificato in anticipo, costituisce un vero spartito musicale aperto e può comprendere spazi pubblici, domestici e privatizzati, percorribili dai pedoni senza compromettere la sicurezza dei partecipanti e in alcuni casi invadere la privacy dei residenti. Attraverso il movimento, il gruppo può interpretare gli eventi acustici urbani spazio-temporali e giocare anche un ruolo nella formazione di essi. Una sorgente sonora come può essere un edificio, inizialmente incontrata proveniente da un aereo, può essere sperimentata da varie posizioni, spostandosi da un piano all'altro in linea con la sorgente stessa, e viceversa. Una fonte sonora come un allarme può essere aumentata poiché si ripercuote in tutti i meandri della città per produrre una sensazione di avvolgimento. Generalmente, questi momenti inconsueti della serendipità uditiva vengono trascurati dalla nostra coscienza, attraverso una convergenza di punti, linee e piani che sono spazialmente differenziati, aderendo così a relazioni simultanee di ritmo, melodia e armonia. Le transizioni da strade strette e riverberanti, racchiuse da facciate di vetro parallele verso spazi aperti a campo libero, possono fornire momenti di tranquillità e respiro.

Il tragitto può andare alla ricerca di un "progetto urbano consapevole del suono", ugualmente può essere esposto all'imposizione di standard governativi sul controllo del rumore, o stimolato dalla realtà cognitiva e manipolativa del panorama sonoro corporativo. La passeggiata però può sondare il ruolo del suono nel fare e connettersi al genius loci provocando anche problemi di identità e radici poiché il gruppo attraversa e si avvicina a zone di diversità etnica, di classe, religiosa e così via. In una camminata realizzata nella città di Modena nel 2017 con un numeroso gruppo di studenti master le variazioni determinate dallo spazio fisico-architettonico e sociale sono risultate palesi lungo l'itinerario, che prevedeva la partenza nella prima periferia fatta di grandi viali ed edilizia popolare, abitata per lo più da classi lavoratrici, fino ad arrivare al piccolo ma opulento centro storico dove portici, vicoli e piazze erano teatro di dimensioni acustiche sociali mutate radicalmente. 


\section{REAZIONI}

Gli oltre sei anni di pratica, che ha coinvolto nel tempo pubblici molto ibridi, di ogni età ed estrazione, dimostrano che l'ascolto dettagliato praticato durante la camminata è stata una esperienza e un esercizio validi a una presa di contatto con il paesaggio acustico. Durante le discussioni conclusive i partecipanti hanno riconosciuto che la passeggiata ha fornito loro un nuovo modus di scoperta del territorio, non privo di sorprese.

Un partecipante particolarmente euforico ha parlato da una prospettiva edonistica della perfetta similitudine con la composizione sonora cinematografica che offre dettagli infiniti.

Un altro invece ha lamentato l'assalto del rumore della realtà, poiché l'esercizio aveva generato un processo di forte sensibilizzazione ed iperacusia.

Un altro partecipante attento ha trovato particolarmente interessante il cambio di superfici dove aveva camminato, in quanto predisponeva l'orecchio a una maggiore attenzione ai dettagli acustici dell'ambiente.

Un partecipante ha dichiarato che essere così dentro la realtà lo ha fatto sentire altrove, in una sorta di dislocazione spazio-temporale.

\section{Conclusioni}

Il soundwalking è una pratica sottile, trasformativa, personale e sensibile, ma è anche una esperienza uditiva analitica e territoriale con implicazioni sociali e politiche. A livello esclusivamente acustico, è un perfetto rituale come un concerto di musica o uno spettacolo teatrale che a mio avviso, soprattutto in Italia, può trovare infiniti terreni di gioco. $\mathrm{E}$ un processo in cui è meglio non avere alcuna fretta anche se dei limiti vanno imposti, e i partecipanti dovrebbero essere incoraggiati ad avere un po' di tempo per sedimentare idee, pensieri, e sensazioni dopo la passeggiata. Per i non specialisti del suono, il soundwalking funge da apriporta per la dimensione acustica della città e aiuta a ridare priorità all'ascolto nel ricco e complesso mix del design urbano e del paradigma in evoluzione dell'urbanistica ambientale. Infine, le esperienze e pratiche artistiche sopra descritte e analizzate costituiscono un importante tassello nello sviluppo della mia ricerca artistica, soprattutto in relazione dell'abitare lo spazio pubblico auspicando prospettive di ascolto sempre più innervate nel tessuto urbano contemporaneo, ricco di complessità da scardinare $\mathrm{e}$ luoghi da riscrivere, ora più che mai. 


\section{RIFERIMENTI BIBLIOGRAFICI}

Caccuri, V. 2015. "Silent Walk". In Sound Development City Artist Expedition '14 Documentation, edited by Dutscha Kistler, 45-59. Zurich: SDC.

Kelly, C., ed. 2012. Sound (Whitechapel Document of Contemporary Art). Cambridge: MIT Press. London: Whitechapel Gallery.

Kotz, L. 2009. "Max Neuhaus: Sound into Space”. In Times Square, Time Piece Beacon, edited by Lynne Cooke, Karen Kelley and Barbara Schröder, 93-111. New Haven: Yale University Press.

Schafer, M.R. 1970. The Book of Noise. Wellington, New Zealand/Vancouver, Canada: Price Milburn \& Co Ltd., P.O. Box 2919/Price Printing LTD.

Senesi, E. 2010. Sonic Rendezvous: Experiences of Urban Sound Art \& Public Encounters in Shared Acoustic Spaces. MA diss., University of Brighton UK.

\section{Sitografia}

http://elisabettasenesi.me/walks.html 